\title{
Development of an Ex Vivo Organ Culture Technique to Evaluate Probiotic Utilization in IBD
}

\author{
Cristiano Pagnini, MD, PhD, Michela Martorelli, MLT, Claudio Lanini, PhD, \\ and Gianfranco Delle Fave, MD
}

\begin{abstract}
The consistent technical and conceptual progress in the study of the microbiota has led novel impulse to the research for therapeutical application of probiotic bacteria in human pathologies, such as inflammatory bowel disease (IBD). Considering the heterogenous results of probiotics in clinical studies, the model of translational medicine may lead to a more specific and efficacious utilization of probiotic bacteria in IBD. In this regard, the selection and utilization of appropriate experimental models may drive the transition from pure in vitro systems to practical clinical application. We developed a simple and reproducible ex vivo organ culture method with potential utilization for the evaluation of probiotic bacteria efficacy in IBD patients.
\end{abstract}

Key Words: probiotic, inflammatory bowel disease, experimental model, organ culture, translational medicine, adhesion

( Clin Gastroenterol 2016;50:S179-S182)

$P^{r}$ robiotic bacteria have been largely proposed in different pathologic conditions and their use has increased in the last decades. ${ }^{1}$ Nonetheless, solid evidence for their use in specific pathologic conditions is limited, and too often their utilization is much more driven by commercial suggestion than by scientific proofs. Recently, probiotic bacteria have been proposed in the setting of inflammatory bowel diseases (IBD). ${ }^{2}$ Indeed, the increased comprehension of the complex pathogenesis of the disease, together with the growing knowledge of the microbiota composition and functions, highly stimulated by the novel culture-independent genomic-based techniques (ie, next-generation sequencing), have consistently pushed forward the idea of the influence of the microbiota in IBD onset and development. ${ }^{3}$ In fact, experimental and clinical evidence that microflora alteration may not only be a consequence of inflammation, but may have an important causative role in IBD course, are mounting. ${ }^{4,5}$ Those findings are stimulating the research on potential therapeutical application of microbiota manipulation, by reduction of potential proinflammatory bacterial species (by antibiotics), stimulation of endogenous bacteria with anti-inflammatory properties (by prebiotics), administration of specific bacteria with favorable profile (probiotics) or of their products (postbiotics), or, lately, with the transfer of the whole fecal echosystem from a healthy donor (fecal transplantation).

From the Faculty of Medicine and Psychology, "Sapienza" University of Rome, S. Andrea Hospital, Rome, Italy.

The authors declare that they have nothing to disclose.

Address correspondence to: Cristiano Pagnini, MD, PhD, Faculty of

Medicine and Psychology, "Sapienza" University of Rome, S.

Andrea Hospital, Via di Grottarossa 1035, Rome 00189, Italy

(e-mail: cristiano.pagnini@uniroma1.it).

Copyright (C) 2016 Wolters Kluwer Health, Inc. All rights reserved. DOI: $10.1097 /$ MCG.0000000000000698
In IBD, the therapeutic application of probiotic bacteria has been so far more convincing in experimental models than in clinical setting. ${ }^{6}$ The reason probably resides primarily in the complexity of human disease, which is not easily reproducible in experimental models. Consequently, animal and experimental models may be at best representative of specific aspects of the human condition, which is characterized by a complex interplay of genetic and environmental factors, as well as by the involvement of different molecular pathways. In the simplification of an experimental model, many therapeutic approaches may prove to be efficacious but may not be directly applicable in clinical setting. Moreover, even though clinical studies of probiotics application in IBD are growing, they are still characterized by a consistent heterogeneity in the design, bacterial species used, dosages, time of treatment, and endpoints. For the design of future trial, the correct selection of the probiotic bacteria to be tested seems to be of particular relevance, as a bacterial species may present peculiar characteristics that may suggest the application for therapeutic purposes only in a specific disease.

Indeed, the rational and scrupulous development of combined in vitro/in vivo studies seems crucial for the proposal and implementation of probiotic bacteria application as therapeutic option in specific pathologic condition, including IBD. In this setting of translational medicine, the role of appropriate "transitional" models (ie, experimental models more representative of the in vivo situation than the traditional in vitro systems, which may favor and guide the transition to a specific clinical application in human) could be of great relevance. In accordance with that, we developed a simple and reproducible ex vivo experimental model to evaluate the adhesion and the mucosal effect of bacterial species administration on normal and pathologic intestinal mucosa.

\section{EX VIVO ORGAN CULTURE EXPERIMENTAL MODEL}

A synthetic scheme of the procedure of the method is represented in Figure 1. Bioptic samples are collected during colonoscopy from normal (proximal and distal segment) and pathologic colon (ie, adenomatous polyps, ulcerative colitis patients). Biopsies are then washed 2 times in fresh phosphate buffered saline (PBS) solution and weighed to avoid consistent difference among samples. For the evaluation of bacterial adhesion, bioptic specimens are put in a $2-\mathrm{mL}$ Falcon tube with $200 \mu \mathrm{L}$ of Roswell Park Memorial Institute (RPMI) media, with the addition of $20 \mu \mathrm{L}(1: 10)$ of a solution of different probiotic formulations in PBS. As a negative control, biotic samples are put in RPMI media with 1:10 of PBS without any probiotic bacteria. The reaction is incubated at $37^{\circ}$ for 2 hours, and 


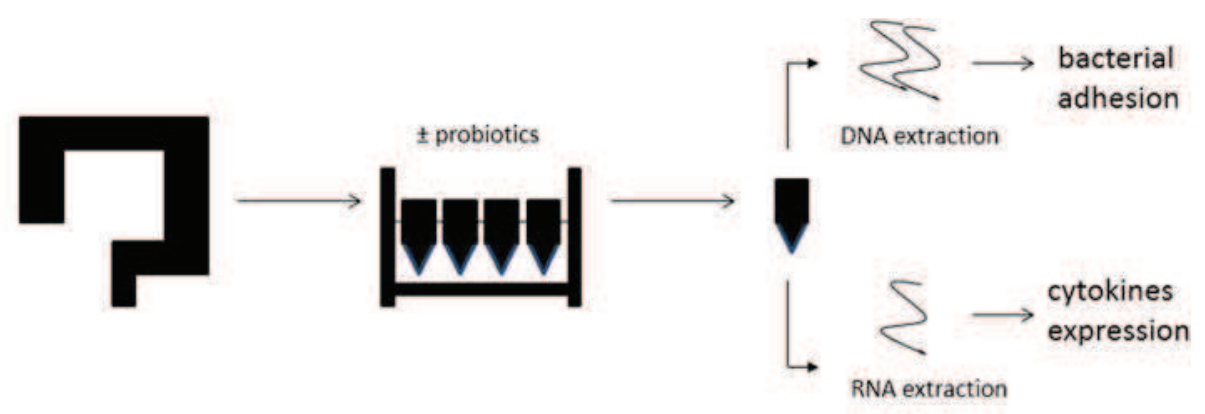

1- colon biopsies collection $\quad 2$-incubation

3 - processing

FIGURE 1. A schematic representation of the ex vivo organ culture technique. For a detailed description refer to the text. full color

then the bioptic samples are collected and washed 2 times in fresh PBS to remove nonadherent bacteria, and finally put in RNA Later solution (Qiagen, Valencia, CA) until further processing. Total DNA is extracted by Qiamp DNA mini kit (Qiagen) according to manufacturer instruction. All the DNA samples are checked by spectrophotometer Gene Quant pro RNA/DNA calculator (Amersham Pharmacia Biotech, Piscataway, NJ) for concentration and purity, and the solutions are brought at the same concentration. Realtime PCR is performed with specific primers for the different probiotics species (already described in the literature), and adherent probiotic bacteria are quantified in a semiquantitative manner by a relative ratio to the lowest detected sample. PCR reactions are performed in a total volume of $20 \mu \mathrm{L}$ in an iCycleriQ detection system (Bio-Rad Laboratories Inc., Hercules, CA), with $16 \mu \mathrm{L}$ of SYBR Green PCR Supermix (Bio-Rad Laboratories Inc.) and $4 \mu \mathrm{L}$ of target DNA. For every set of primers, the original cycle conditions are generally maintained. House-keeping genes ( $\beta$-actin and GADPH) were used for normalization of the values. After reactions, PCR products are run on a $2 \%$ agarose gel with ethidium-bromide staining for visualization.

For the evaluation of mucosal effect of probiotic bacteria, we apply the same model to test the expression of proinflammatory and anti-inflammatory cytokines, with few variations. In particular, bioptic samples are incubated for a longer time $(6 \mathrm{~h})$ to evaluate variation in mRNA production after the incubation, and we add to the RPMI media with bioptic samples a 1:10 solution of probiotic conditioned media, prepared according to a procedure already described in the literature, ${ }^{7}$ or PBS alone for negative controls. We set the incubation time to 6 hours as longer time resulted in contamination of the reaction and degradation of RNA, with a consistent reduction of expression of the house-keeping genes. After incubation, total RNA is extracted by RNAeasy miniprep kit (Qiagen), cDNA synthesized by GeneAmp RNA PCR kit (Applied Biosystems, Foster City, CA), and mRNA for specific cytokines quantified by real-time PCR and normalized to house-keeping gene concentration, as already described above.

\section{PRELIMINARY RESULTS: PROBIOTIC MUCOSAL ADHESION}

To test mucosal adherence of different probiotic bacteria, mucosal biopsies from normal proximal and distal colon, taken from 6 subjects in whom colonoscopy did not show macroscopic or microscopic evidence of pathology, were incubated, according to the aforementioned described protocol, with a commercially available multispecies probiotic compound. Analyzing the mucosal adhesion of 3 representative bacteria of the formulation, we observed that different bacterial species adhered in a peculiar manner to the proximal and the distal colonic specimens. In particular, after incubation, a consistent adhesion of Streptococcus thermophilus and Bifidobacterium infantis was observed, whereas Lactobacillus acidophilus was not detectable either in the proximal or in the distal colonic segments. Considering relative segmental adhesion, we found a significantly higher adhesion of $B$. infantis to the proximal compared with the distal specimens, and a similar trend for $S$. thermophilus, even though to a lesser degree (Fig. 2). Those data support the hypothesis that probiotic species may adhere in a peculiar way to the intestinal mucosa, and an experimental model that can assess that property could be of great relevance for the specific selection of bacterial species for utilization in pathologic conditions.

\section{DISCUSSION}

The investigation of probiotic bacteria in clinical settings has been influenced by the technical and conceptual revolution of the microbiota field. Nowadays, 2 different approaches are leading the research of possible clinical application of probiotics. On one hand, the accurate analysis of microbiota alteration in pathologic versus physiological conditions, made possible by the novel sequencing techniques available, is leading to the identification, and to the possible test, of bacterial species with a possible specific role in the prevention of the pathologic conditions investigated. However, the fascinating approach of the development of those novel probiotic species has several limitations. In fact, different composition in microbiota between patients and normal controls may not necessarily imply a causative role of the bacteria for the pathologic condition. Moreover, as the putative bacteria have not been usually used as a supplement in humans before, the identified species would need a thorough in vitro and in vivo characterization in terms of safety, efficacy, and stability both in drug formulation and in the gastrointestinal tract. On the other hand, the other branch of research is focusing on the in-deep characterization of the properties of probiotic bacteria already known and diffuse in the market, 
A

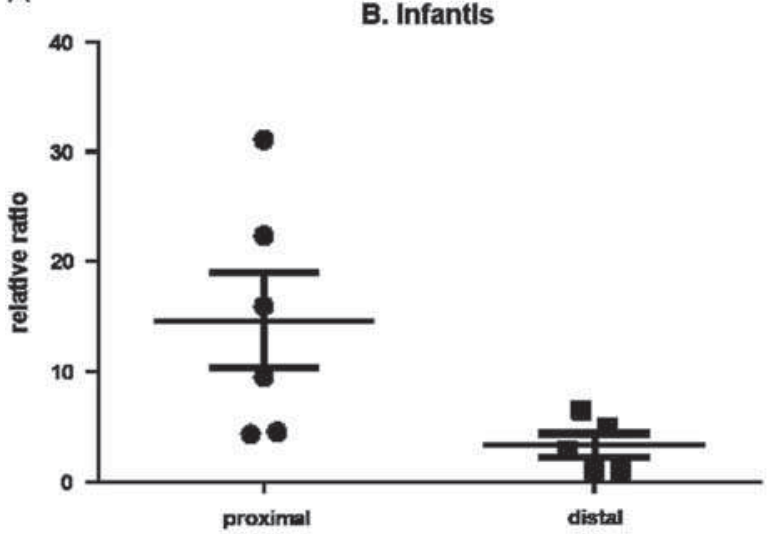

B

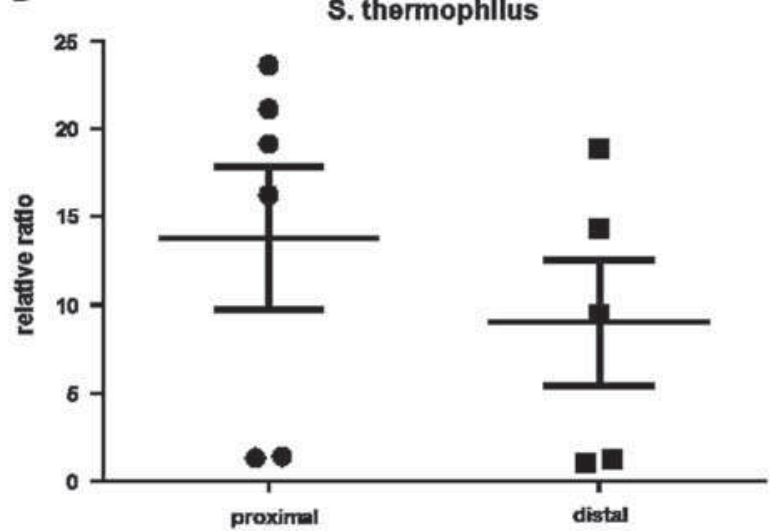

C

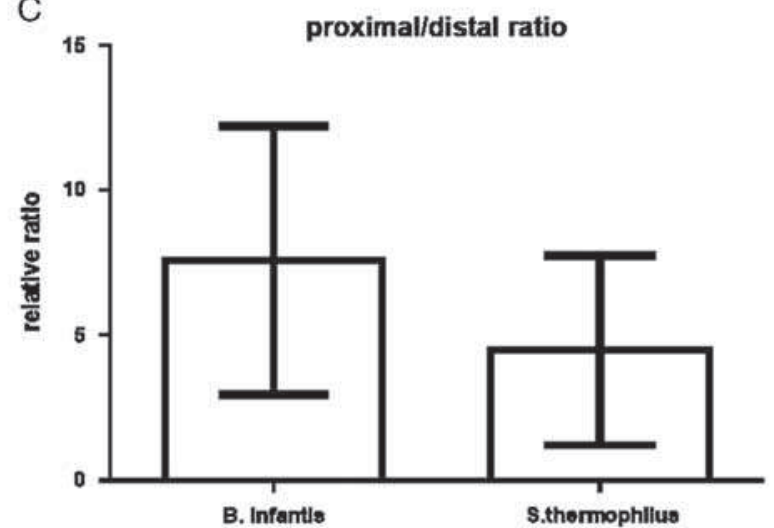

FIGURE 2. A and B, Mucosal DNA concentration of Bifidobacterium infantis and Streptococcus thermophilus in the proximal and the distal colonic biopsies after incubation with multiple probiotic compound. Lactobacillus acidophilus was not detectable. Data are expressed as a relative ratio to the lowest detectable sample. $C$, Relative increment in proximal versus distal colonic specimens in the single individual.

such as L. rhamnosus GG, which represents the most studied species in this class of bacteria. In this setting, the aim of the research is to evaluate specific characteristic of the bacteria with a potential application in particular clinical situation, with the advantage of the major comprehension of the general characteristics and of the safety profile of the bacteria. In fact, as those bacteria are already in use from quite a long time, drug formulations have been already developed and the safety profile is generally favorable. Nonetheless, utilization of such products is often empirical and unspecific, and appropriate evaluation in specific clinical indication is warranted. In both methods of research, the preclinical evaluation and selection of specific probiotic species with specific features may increase and lead the utilization of such bacteria in appropriate clinical settings.

For this purpose, we developed and described an ex vivo organ culture experimental model. The present method has not been directly validated so far, but, for the evaluation of bacterial adhesion, indirect proof of correlation with the in vivo adhesion is available. In fact, we have previously demonstrated an in vivo reduction of concentration of total mucosal adherent bacteria in adenomatous polyps compared with adjacent normal mucosa, together with an increment of production of antibacterial molecules, such as $\alpha$-defensins. A similar reduction of bacterial concentration in polyp mucosa was observed in the ex vivo experimental model, when bioptic samples were incubated with a multistrain probiotic formulation. ${ }^{8}$ Moreover, a similar pattern of adhesion of the 3 bacteria, tested in the present paper in normal human colon, was observed in vivo in a mouse model of spontaneous ileitis (ie, SAMP1/YitFc). In fact, after 6 weeks of supplementation at high dose with the multiple probiotic formulation, only $S$. thermophilus and $B$. infantis, but not $L$. acidophilus, were detectable at mucosal level in the terminal ileum of mice, despite the latter bacteria being consistently detected in DNA extracted from feces. ${ }^{9}$

The potential usefulness of the model for the evaluation of mucosal effect of the tested bacteria remains to be determined, and comparison with experimental and in vivo findings for cytokines expression after probiotic administration are currently ongoing. One could speculate that, as in the ex vivo model the bioptic samples are simply put in incubation with probiotic conditioned media, direct paracellular stimulation of the lamina propria compartment by probiotic media may occur. For that reason, the model may not be fully representative of the in vivo situation, where the epithelial cells represent the main interface between luminal content and subepithelial compartment. To address that point, comparison with the effect of probiotic on cells of the different intestinal compartments may help in the interpretation of data from the experimental model. To better mimic the in vivo situation, an experimental model in 
which surgical specimens are cultured and polarized has been recently described. ${ }^{10}$ Compared with the model described in the present paper, such a model offers the advantage of a more detailed study of the immune mucosal effect of different stimuli (ie, different probiotic bacteria), in particular for the utilization of a cave cylinder that delimited the area of stimulation on the apical face of the mucosa. In contrast, such a model is basically more complex and necessitates surgical mucosal explants that are less easily available compared with bioptic specimens.

\section{CONCLUSIONS}

In the present paper, we have described an experimental method that is potentially applicable in every laboratory, which do not need complex procedures and is of limited cost. The application and the potential limitation of this experimental model are still to be fully explored, but with that simple and reproducible method the adhesion of different bacterial species to normal and pathological colon may be tested. As different probiotic species adhere in a peculiar way to normal and pathological colon, as well as in different segments of the normal colon, such a method may be useful for the selection of bacteria for the treatment of specific pathologic situation. For instance, probiotic bacteria with an elective adhesion to the distal colon may be particularly indicated in the distal ulcerative colitis, whereas a species with a widespread adhesion to the whole colon may be more appropriate in pancolitis. Studies for the evaluation of this method for the effect of the bacteria on mucosal cytokines expression and production are ongoing.

The correct application of translational medicine, with the appropriate utilization of specific experimental models for the selection of bacterial species with well-described characteristics for specific clinical situation, will hopefully lead to a more tailored and efficacious utilization of probiotic bacteria in the field of IBD treatment.

\section{REFERENCES}

1. Guarner F, Khan AG, Garisch J, et al. World Gastroenterology Organisation Global Guidelines: probiotics and prebiotics October 2011. J Clin Gastroenterol. 2012;46: 468-481.

2. Pagnini C, Delle Fave G, Bamias G. Probiotics in inflammatory bowel disease: pathophysiological background and clinical applications. World J Immunol. 2013;3:31-43.

3. Preidis GA, Versalovic J. Targeting the human microbiome with antibiotics, probiotics, and prebiotics: gastroenterology enters the metagenomics era. Gastroenterology. 2009;136: 2015-2031.

4. Garrett WS, Lord GM, Punit S, et al. Communicable ulcerative colitis induced by T-bet deficiency in the innate immune system. Cell. 2007;131:33-45.

5. Moayyedi P, Surette MG, Kim PT, et al. Fecal microbiota transplantation induces remission in patients with active ulcerative colitis in a randomized controlled trial. Gastroenterology. 2015;149:102-109 e106.

6. Claes IJ, De Keersmaecker SC, Vanderleyden J, et al. Lessons from probiotic-host interaction studies in murine models of experimental colitis. Mol Nutr Food Res. 2011;55:1441-1453.

7. Petrof EO, Kojima K, Ropeleski MJ, et al. Probiotics inhibit nuclear factor-kappaB and induce heat shock proteins in colonic epithelial cells through proteasome inhibition. Gastroenterology. 2004;127:1474-1487.

8. Pagnini C, Corleto VD, Mangoni ML, et al. Alteration of local microflora and alpha-defensins hyper-production in colonic adenoma mucosa. J Clin Gastroenterol. 2011;45:602-610.

9. Pagnini C, Saeed R, Bamias G, et al. Probiotics promote gut health through stimulation of epithelial innate immunity. Proc Natl Acad Sci U S A. 2010;107:454 459.

10. Tsilingiri K, Sonzogni A, Caprioli F, et al. A novel method for the culture and polarized stimulation of human intestinal mucosa explants. J Vis Exp. 2013;75:e4368. 